\title{
Superconducting properties of niobium after electron beam welding
}

\author{
Prakash N. Potukuchi, ${ }^{1}$ L. S. Sharath Chandra, ${ }^{2}$ M. K. Chattopadhyay, ${ }^{2}$ D. Kanjilal, ${ }^{1}$ Amit Roy, ${ }^{1}$ and S. B. Roy ${ }^{2}$ \\ ${ }^{1}$ Inter-University Accelerator Centre, Aruna Asaf Ali Marg, New Delhi-110067, India \\ ${ }^{2}$ Magnetic and Superconducting Materials Section, Raja Ramanna Centre for Advanced Technology, Indore-452013, India
}

(Received 9 May 2011; published 1 December 2011)

\begin{abstract}
One of the major criteria for designing superconducting niobium resonant cavities is to minimize the peak surface electric and magnetic fields to maximize the achievable accelerating electric gradient. Even after addressing the extrinsic effects adequately, a large number of cavities perform below the theoretical gradient limit. The peak magnetic field for the first flux-line penetration in the superconducting state of niobium, which either severely degrades the cavity quality factor or results in complete thermal breakdown, is an important limitation. The flux-line penetration is known to depend on the microstructural properties of niobium which may get altered in the process of cavity fabrication. The most common technique of fabricating niobium cavities is to form their components using standard sheet metal techniques and join them by electron beam welding in vacuum. We present results of a study on the superconducting response through magnetization measurements in the electron beam welded region of niobium to understand the limitations (if any) posed by the welding in achieving the highest gradient. We also present and discuss results on the performance of niobium quarter wave resonators incorporating such electron beam welds in the high magnetic field region.
\end{abstract}

DOI: 10.1103/PhysRevSTAB.14.122001

PACS numbers: 29.20.Ej, 74.25.Ha, 74.25.Op, 74.70.Ad

\section{INTRODUCTION}

Superconducting niobium resonant cavities are used in a wide range of modern accelerators for accelerating electrons, protons, and heavy ions. The low velocity TEM class resonators are used for accelerating heavy ions [1], although they are also being increasingly designed for accelerating protons [2]. They are optimized for the wide range of ion velocities $\beta(=v / c)=0.009-0.63$ and operate in the frequency range of 50-350 MHz. At these frequencies the Bardeen Cooper Schrieffer (BCS) surface resistance of superconducting niobium is small enough to permit their operation at $4.2 \mathrm{~K}$ with a high quality factor $(Q)$. The medium and high velocity TM class cavities are generally used for accelerating electrons and protons. They are designed for particle velocities $\beta=0.5$ all the way up to the speed of light and operate in the frequency range 350-3000 MHz. To reduce the BCS surface resistance of superconducting niobium at these frequencies to an economically tolerable value, the TM class cavities are operated at $2 \mathrm{~K}$. Several variants of the TEM and TM class structures have been designed and employed in particle accelerators [3-5].

Achieving high accelerating gradients in resonant cavities is the prime concern of cavity designers. Resonant cavities are always designed to minimize the peak electric field, $E_{\text {peak }}$, and the peak magnetic field, $B_{\text {peak }}$, inside the

Published by the American Physical Society under the terms of the Creative Commons Attribution 3.0 License. Further distribution of this work must maintain attribution to the author(s) and the published article's title, journal citation, and DOI. cavity, to maximize the accelerating electric gradient $E_{a}$. The ultimate achievable accelerating gradient is limited by the critical magnetic field above which the superconducting material ceases to remain in the superconducting Meissner state. Hence, $B_{\text {peak }}$ needs to remain below this critical magnetic field. However, several extrinsic effects, such as surface defects, impurities, resonant electron multipacting, and field emission, can limit the achievable accelerating gradient in a superconducting radio frequency (SCRF) cavity to a value well below the intrinsic limit set by the critical magnetic field. In the past two decades, much effort has gone into addressing these extrinsic issues very successfully. Many techniques, including eddy current scanning of niobium sheets [6], high pressure rinsing of the cavities [7], final assembly in class-100 or better clean rooms, and $120^{\circ} \mathrm{C}$ baking [8], have greatly reduced their effect and helped in pushing the accelerating gradient up. However, a large number of cavities perform below their ultimate intrinsic gradient limit.

Niobium is a type-II superconductor. For niobium SCRF cavities the critical magnetic field leading to the intrinsic gradient limit is related to the lower critical magnetic field $H_{C 1}$ of superconducting niobium [4]. The value of $H_{C 1}$ for pure niobium material is $\sim 1800$ Oe at $2 \mathrm{~K}$ and $\sim 1400$ Oe at $4 \mathrm{~K}[9,10]$. In a type-II superconductor, it is expected that the Meissner state may continue to exist as a metastable state in fields higher than $H_{C 1}$ up to the thermodynamical critical field $H_{C}$ (or the superheating field $H_{\mathrm{SH}}$ ). In the SCRF literature it is often assumed that the intrinsic gradient limit is this superheating field $H_{\mathrm{SH}}$. This assumption is mainly based on the argument that the period of the rf field in an SCRF cavity is shorter than the nucleation 
time of Abrikosov mixed state [11]. This view needs to be considered with a certain degree of caution. First, sustaining the metastable superheated (supercooled) state across a first order phase transition is possible only in the case of an ultrapure sample without any defects [12]. Even in such cases the height of the energy barrier separating the equilibrium state and the metastable state decreases steadily as the limit of superheating (supercooling) is approached. At the superheating limit any infinitesimal energy fluctuation can drive the metastable state to the stable state [12]. Any defect/disorder will reduce the height of this energy barrier drastically at the site of the defect/disorder and encourage the formation of the stable state. In addition, the oscillating rf field will be a source of small energy fluctuations, which is detrimental to the existence of the metastable state. High purity superconducting niobium materials, characterized by high residual resistivity ratio (RRR), are usually used for fabricating SCRF cavities. Even in such materials there will be a finite amount of defects or disorders, which may further increase during the cavity fabrication process. Thus, for SCRF cavities the lower critical field $H_{C 1}$ is a better design parameter than $H_{\mathrm{SH}}$. In superconducting materials with finite size and shape, the magnetic field can actually enter inside the material at an applied field $H_{P}$, which is even lower than $H_{C 1} . H_{P}$ is designated as the field at which the magnetic flux lines first penetrate inside the superconducting material [10]. In a defect-free type-II superconducting material with ideal sample geometry, $H_{P}$ coincides with $H_{C 1}$. However, for geometries different from that of an infinitely long thin sample, $H_{P}$ is expected to be perceptibly different than $H_{C 1}$. This matter has been highlighted recently in the context of technical niobium samples used in SCRF cavity fabrication [10].

The limitation on the achievable accelerating gradient due to magnetic field was demonstrated in an experiment carried out on a single cell niobium cavity operating in $\mathrm{TE}_{011}$ mode, which has no electric field on the surface [13]. $Q$ degradation at high gradients was observed in this cavity, suggesting the possibility of the involvement of surface magnetic fields for the rf losses. The critical magnetic field at which the $Q$ degradation is observed in the single cell niobium cavity operating at $2 \mathrm{~K}$ is in the range of 1000-1200 Oe [13]. This is in contrast with the value of $H_{C 1} \sim 1800$ Oe for pure niobium material at $2 \mathrm{~K}[9,10]$. The results from this experiment were further substantiated in a recent study which looked at the influence of buffered chemical treatment on the field for first flux-line penetration $H_{P}$ in niobium [14]. It indicated that buffered chemical polishing (BCP) substantially lowered the value of $H_{P}$ at $2 \mathrm{~K}$ to around $1000-1100 \mathrm{Oe}$. The superior performance of electropolished cavities over BCP cavities has been demonstrated quite conclusively [15]. A separate study on surface superconductivity indicated that electropolishing (EP) offered a superior alternative to BCP in terms of achieving higher gradients in resonant cavities [16].
The most common method of fabricating niobium cavities is to form their components using standard sheet metal techniques and join them by electron beam welding (EBW) in vacuum. In this method of fabrication it is not possible to avoid electron beam welding joints in the high magnetic field regions of the TM class cavities as well as the TEM class resonators. The aim of the present study is to find whether the process of EBW in any way influences the superconducting properties of niobium materials which could ultimately influence the performance of a SCRF cavity. A systematic study of superconducting properties (including the field for first flux-line penetration $H_{P}$ ) through magnetization measurements of the welded region of niobium will therefore be important for understanding the limitations (if any) arising from the process of EBW in achieving the highest gradient in a niobium SCRF cavity. Another important issue is to identify if one type of EBW joint design is more preferable than the other.

\section{EBW JOINT DESIGNS}

The EBW joints in cavities are usually designed in two different configurations, namely, square butt joint and step joint. In Fig. 1, the cross sections of both joint designs are shown. Butt joints are used in the seam welding of cylinders and saddles where machining a step is neither possible nor required. Step joints are predominantly used where it is important to avoid radial displacement in mating components, e.g., in joining two cylinders. Occasionally it is possible to avoid a step joint by designing appropriate fixturing. However, in some situations it is not possible to avoid it. For example, the coaxial line to the shorting plate joint and the shorting plate to the outer cylinder joint in a quarter wave resonator (QWR), such as the ones used in the superconducting linac at Inter-University Accelerator Centre (IUAC) shown in Fig. 2, are likely to be step joints. But this is also the region where the magnetic field in the resonator reaches its maximum value. In an elliptical cavity operating in the $\mathrm{TM}_{010}$ mode, the electron beam welding joint is in the high magnetic field region of the equator and the joint may be butt or step type.

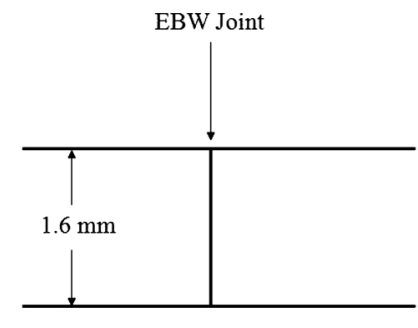

(a)

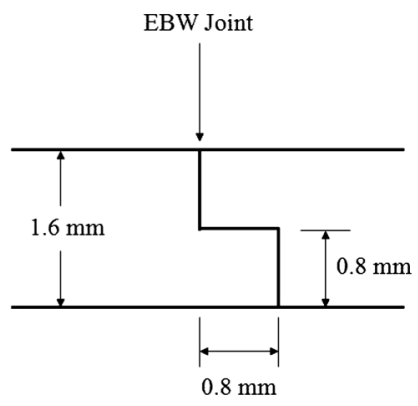

(b)
FIG. 1. Cross section of (a) butt joint design and (b) typical step joint design. 


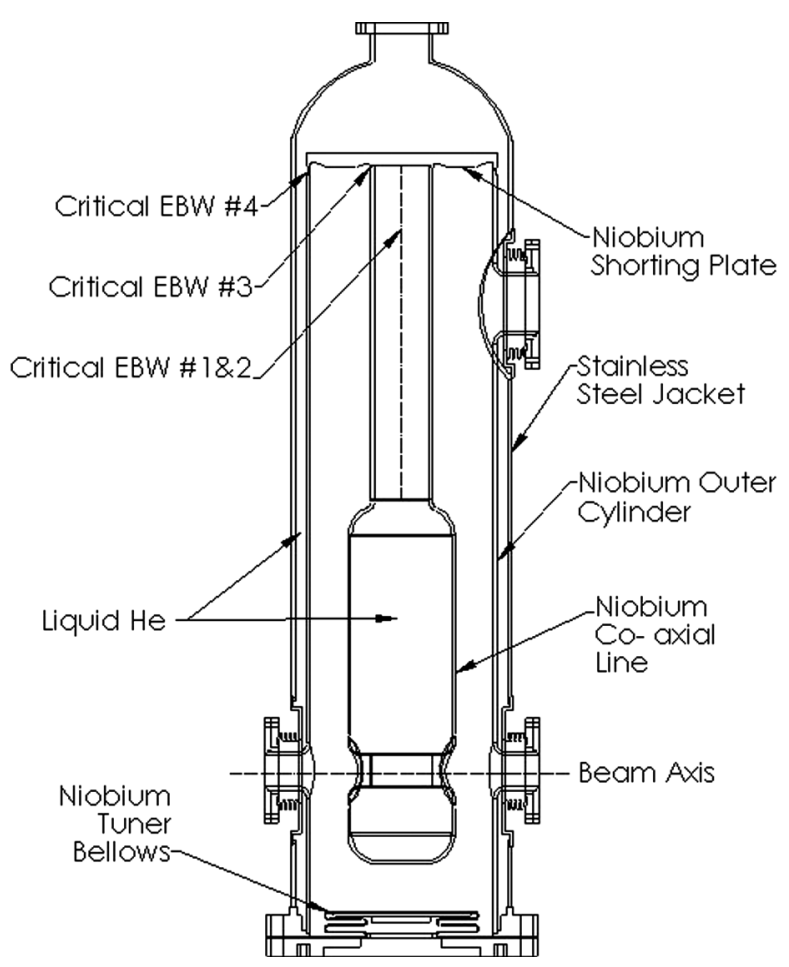

FIG. 2. Schematic diagram of the IUAC QWR. The overall height of the resonator is about $80 \mathrm{~cm}$. The critical welds in the high magnetic field region of the resonator are indicated. Welds \#1 and 2 are long seam butt joints on the upper section of the coaxial line; the first weld is on the front side and the second weld is on the back side. Welds \#3 and 4 are both step joints.

Butt joints are easier to machine and clean as compared to step joints. The total machined area in a step joint is $50 \%$ more compared to a butt joint of the same thickness. It is reasonable to assume that contamination in the machined area may therefore proportionately go up in a step joint compared to a butt joint.

In the following sections, experimental details and results of the measurements of superconducting properties made on electron beam welded butt and step joints of niobium materials are presented.

\section{NIOBIUM SAMPLE PREPARATION}

The niobium samples for the present measurements were prepared with great care by following the same procedures used for making typical cavity components. The niobium material procured from Wah Chang, USA had: (1) average grain size of $50 \mu \mathrm{m}$ with typical tantalum content of about $300 \mathrm{ppm}$, and (2) RRR value of around 300. The butt and step joints were made out of niobium of thicknesses $1.6 \mathrm{~mm}$ (see Fig. 1), which is close to the thickness of niobium used for making critical welding joints in high magnetic field regions of the cavities, e.g., $1.6 \mathrm{~mm}$ in the shorting plate to outer cylinder joint in the quarter wave resonators (Fig. 2) and $1.7 \mathrm{~mm}$ in the equator joint of the TESLA-type cavities. Each sample was approximately $100 \mathrm{~mm}$ wide and 150-200 $\mathrm{mm}$ long. They were machined using the same tools, cutting speeds, coolants, and fixtures which were used for making actual cavity components. The post-machining deburring of the edges and mechanical polishing of the samples was done by hand using files exclusively used for niobium work and $\mathrm{Al}_{2} \mathrm{O}_{3}$ emery paper of varying grades. After machining, the samples were thoroughly cleaned using analytical grade isopropyl alcohol and degreased in an ultrasonic cleaner for approximately one hour. The samples were then chemically etched using a solution of $\mathrm{HNO}_{3}$ of concentration (conc.) $76 \%$, HF (conc. 40\%), and water mixed in the volumetric ratio $2: 2: 1$, to remove about $2-3 \mu \mathrm{m}$ material and rinsed with deionized water (conductivity $18 \mathrm{M} \Omega-\mathrm{cm})$. After drying they were packed in clean dry bags and carried to the electron beam welding facility.

For doing the electron beam welding, the samples were mounted on a fixture (see Fig. 3) made for this purpose. The fixture was designed to keep the niobium shims, used for clamping the samples, sufficiently far away $(18-20 \mathrm{~mm})$ from the heat affected zone. On the bottom side of the fixture (i.e. the side facing the root of the weld), a niobium shim was used to prevent the welded region from getting contaminated (from the evaporation of metal vapor). All the weldings were performed at $<5 \times 10^{-5} \mathrm{mbar}$ pressure. After welding, the samples were left in vacuum for at least one hour for them to cool down below $50^{\circ} \mathrm{C}$ before removing.

After electron beam welding, a surface layer of thickness $\sim 150 \mu \mathrm{m}$ was removed from the niobium materials by electropolishing (EP) [17]. For electropolishing the niobium samples, a mixture of $\mathrm{H}_{2} \mathrm{SO}_{4}$ (conc. 98\%) and HF (conc. 40\%) in the volumetric ratio 17:3 was used. A helical shaped aluminum tube served as the cathode. Water from a separate chiller unit was circulated through the cathode to maintain the acid temperature between 28 and $32^{\circ} \mathrm{C}$. During EP, 18 volts DC was applied for one minute after which the power supply was switched off for one minute. During the off period the acid was recirculated to wash off the oxide layer from the niobium surface. This

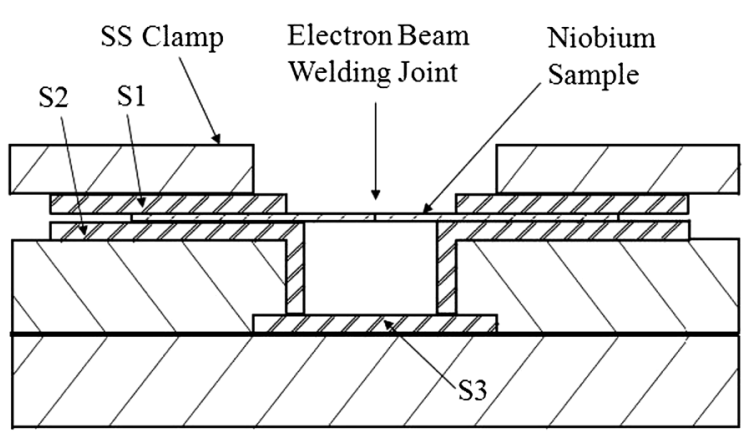

S1, S2, S3: Niobium Shims

FIG. 3. Cross section of the fixture used for preparing the electron beam welded niobium samples. 
process of turning on the power supply for one minute and turning it off for one minute constitutes one cycle. Approximately 20 cycles of EP removes $25 \mu \mathrm{m}$ from the niobium surface. During EP the current drawn from the power supply is $\sim 0.1 \mathrm{~A} / \mathrm{cm}^{2}$. No heat treatment was applied on the samples after electropolishing.

\section{EXPERIMENTAL DETAILS}

Superconducting response of the various niobium samples thus prepared has been studied through DC magnetization measurements. A typical experimental arrangement for magnetization measurements is shown in Fig. 4 where an applied DC field, $H_{\mathrm{DC}}$, magnetizes the sample to a magnetic moment $M$. A detection coil is then used to detect the change in magnetic flux due to the presence of the magnetic moment of the sample. A relative movement between the sample and the detection coil system is necessary in order to vary the sample flux coupled to the detection coil. In a vibrating sample magnetometer (VSM) the sample is vibrated and this vibration generates an $\mathrm{AC}$ signal at a frequency determined by the frequency of vibration. In actual measurements, a system of coils of various geometries is used to minimize the background effect and noise. We have used a commercial VSM (Quantum Design, USA) for our magnetization measurements. The details of the working principle of this magnetometer are available in Ref. [18].

Two different experimental protocols, zero field cooled (ZFC) warming and field cooled cooling (FCC), are used for temperature dependent magnetization measurements. In ZFC mode the sample is cooled to the lowest temperature of measurement before the magnetic field is switched

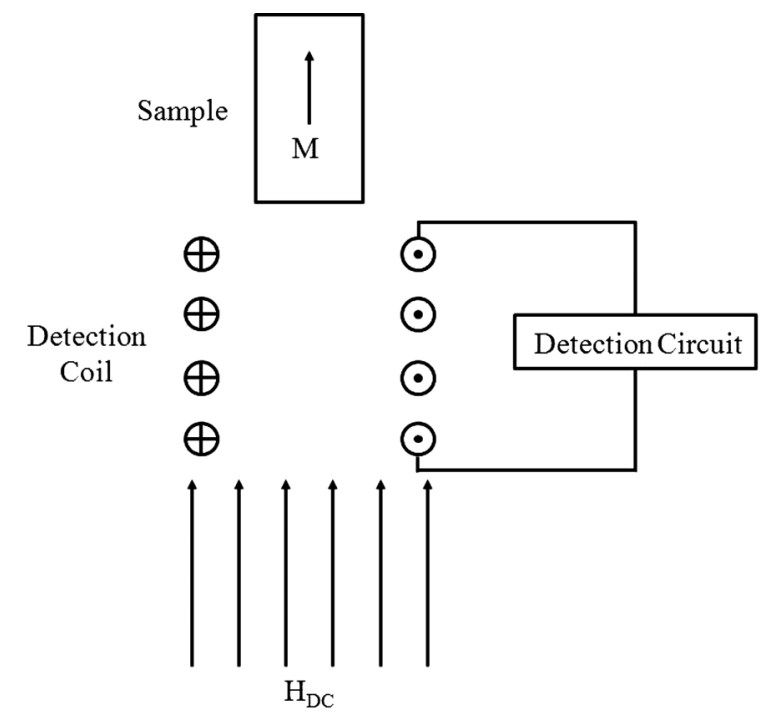

FIG. 4. Schematic diagram of the magnetization measurement setup. For the measurements on the electron beam welded niobium samples, the orientation of the weld bead was parallel to the plane of the paper. on and the measurement is made while warming up the sample. In the FCC mode the applied magnetic field is switched on in the temperature regime well above the superconducting transition temperature $\left(T_{C}\right)$ of the niobium samples and the measurement is made while cooling across the $T_{C}$ to a particular temperature of measurement, say $2 \mathrm{~K}$. A superconducting magnet is used in the VSM to generate the external magnetic field. Such superconducting magnets are likely to have trapped magnetic fields after a field cycle. It is a standard practice to apply a field $H>50$ Oe during a temperature dependent magnetization measurement to minimize the uncertainty related to the trapped field. In the present work we have applied a field of 100 Oe during the temperature dependent studies in the niobium samples.

All the isothermal field dependent magnetization measurements are performed after cooling the sample to the designated temperature of measurements in the ZFC mode. For a uniform comparison of results we have used the same sample dimensions of $\sim 2 \mathrm{~mm} \times 2 \mathrm{~mm} \times 1.6 \mathrm{~mm}$ in our studies. This ensures the same demagnetization factor for all the samples. The small samples were cut from the welded plate using a diamond wheel [19] with a suitable coolant. In the present study we have performed magnetization measurements on multiple samples obtained from various parts of the welded region of both categories (i.e. step joint and butt joint). However, we did not find any significant difference in the results obtained on samples from the different regions. For the sake of conciseness, we report the results of our study on the samples obtained from the midportion of the welded region.

\section{RESULTS AND DISCUSSION}

In Fig. 5 (Fig. 6) we present the magnetization $(M)$ versus temperature $(T)$ plot for electropolished EBW step joint (butt joint) niobium materials obtained in ZFC and

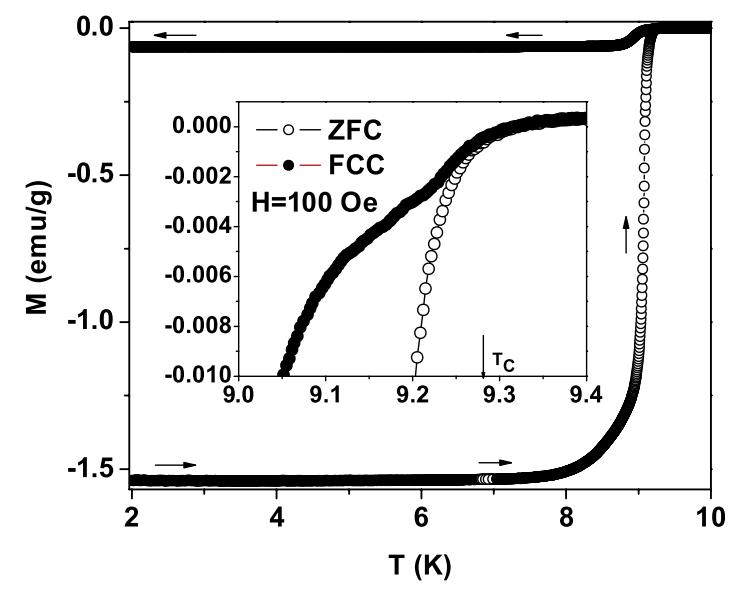

FIG. 5. $\quad M$ versus $T$ plot for electron beam welded and electropolished niobium step joint in an applied field of 100 Oe. The inset highlights the temperature region near $T_{C}$. 


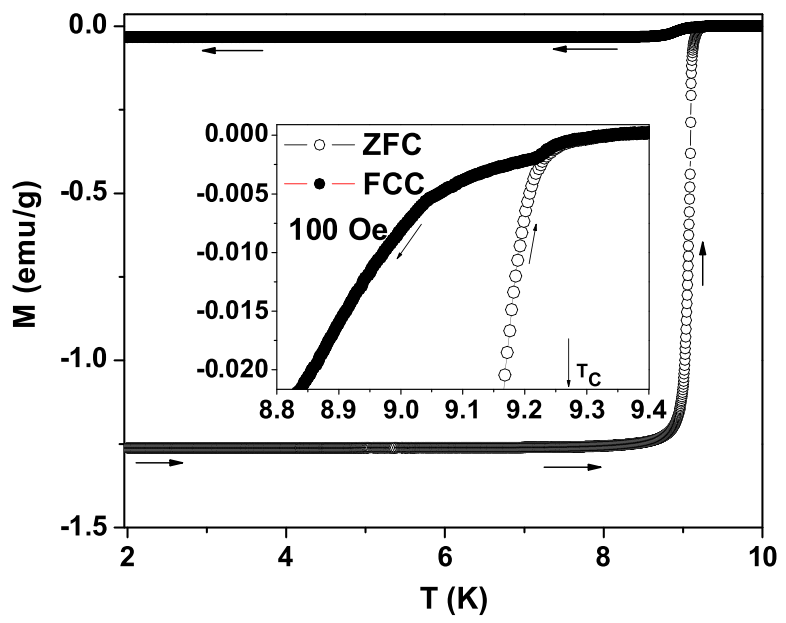

FIG. 6. $\quad M$ versus $T$ plot for electron beam welded and electropolished niobium butt joint in an applied field of 100 Oe. The inset highlights the temperature region near $T_{C}$.

FCC mode in an applied field of 100 Oe. The superconducting transition temperature $\left(T_{C}\right)$ for the EBW step joint and butt joint niobium materials estimated from the onset of diamagnetism are $\sim 9.28$ and $\sim 9.26 \mathrm{~K}$, respectively. We note that the $T_{C}$ estimated here is obtained in the presence of an applied field of $100 \mathrm{Oe}$, and hence expected to be slightly lower than the zero field $T_{C}$. This small difference, however, is negligible for the purpose of the present study. To compare with these results of EBW niobium materials, we plot in Fig. 7, $M$ versus $T$ plot for an electropolished sample of niobium material obtained in ZFC and FCC mode in an applied field of $100 \mathrm{Oe}$. The $T_{C}$ of this electropolished niobium material is $\sim 9.2 \mathrm{~K}$. The estimated $T_{C}$ 's of all these niobium materials (both normal and EBW joints) are very similar to the reported value of $T_{C}$ on good quality niobium materials [9]. These results clearly indicate that the superconducting transition temperature of

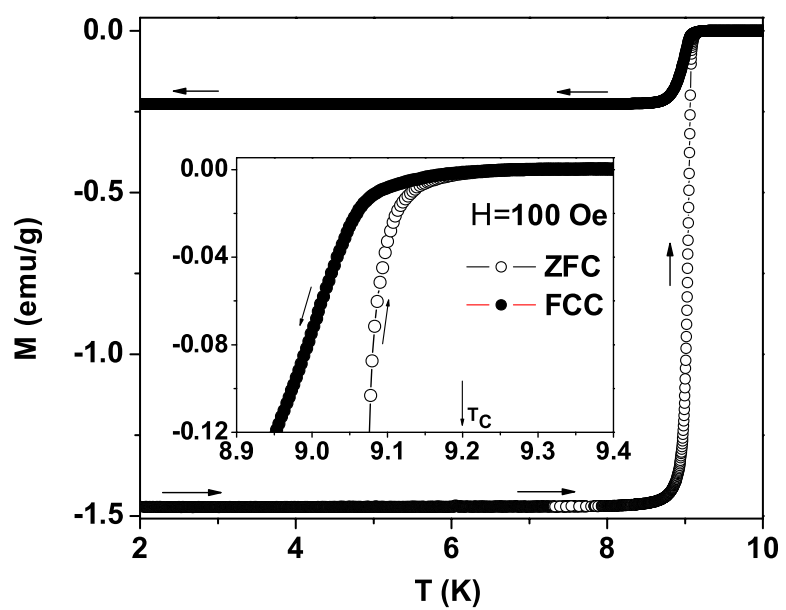

FIG. 7. $M$ versus $T$ plot for electropolished as-received niobium in an applied field of 100 Oe. The inset highlights the temperature region near $T_{C}$. niobium materials is not degraded by the process of EBW. With this encouraging result, we shall now investigate the isothermal magnetic field dependence of superconducting properties of niobium materials with EBW step joint and butt joint. The performance of a niobium SCRF cavity would critically depend on the response of the niobium materials to such magnetic fields (arising out of the applied rf electric field).

Figure 8(a) [Fig. 8(b)] shows the magnetization $(M)$ versus magnetic field $(H)$ plot for electropolished EBW step joint (butt joint) niobium materials, obtained after zero field cooling the samples to $4 \mathrm{~K}$ from a temperature above the $T_{C}$ of the samples. The $M-H$ plots are markedly hysteretic in nature. The observed hysteresis indicates the existence of strong pinning centers for the magnetic flux lines in these samples. In the first approximation, the field for first magnetic flux-line penetration can be estimated as

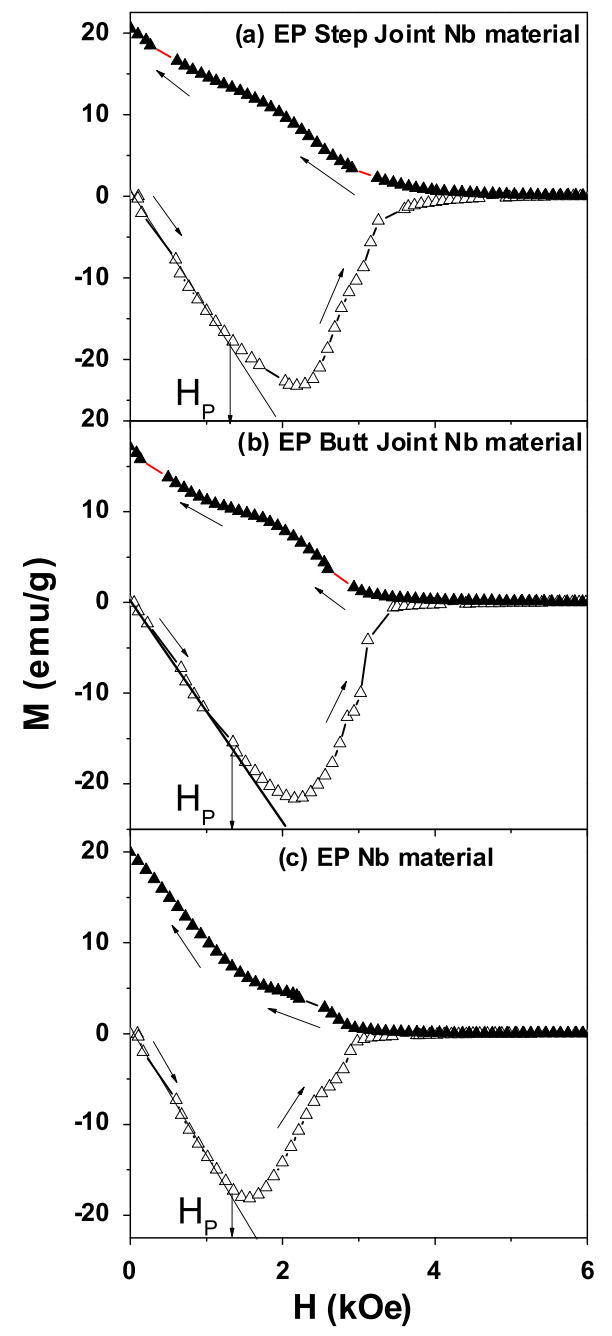

FIG. 8. Isothermal $M$ versus $H$ plots of (a) electron beam welded and electropolished niobium step joint (top), (b) electron beam welded and electropolished niobium butt joint (middle), and (c) electropolished as-received niobium (bottom), starting from the zero field cooled state at $4 \mathrm{~K}$. 
the field $H_{P}$ where the virgin leg of the isothermal $M-H$ curve deviates from the linear $H$ dependence [10]. In a defect free sample of a type-II superconductor with ideal sample geometry, $H_{P}$ would be identical with the lower critical field $H_{C 1}$ and the $M-H$ curve will be perfectly irreversible. The estimated value of $H_{P}$ in all these samples is $\sim 1300$ Oe $(\sim 1350$ Oe) for the step joint (butt joint) niobium sample, which is quite close to the expected value of $H_{C 1} \sim 1400 \mathrm{Oe}$ (at $T=4 \mathrm{~K}$ ) in good quality samples of niobium [9]. This statement is further supported by the measured isothermal $M-H$ plot of an electropolished sample of niobium material (as received from vendor) at $4 \mathrm{~K}$ [see Fig. 8(c)]. A comparison of Figs. 8(a)-8(c) clearly shows that the EBW process did not affect the expected magnetic field response of the superconducting state including $H_{P}$ of niobium materials. From these studies of step and butt joint niobium materials, one cannot really distinguish the relative merit of these two processes with respect to each other as far as superconducting properties (especially the intrinsic limit of the accelerating gradient) are concerned.

Since the TM class SCRF cavities usually operate at $2 \mathrm{~K}$, it is worth investigating the superconducting properties of the welded region of niobium at $T=2 \mathrm{~K}$. For this purpose we present in Fig. 9(a) [Fig. 9(b)] the magnetization (M) versus magnetic field $(H)$ plot for electropolished EBW step joint (butt joint) niobium material, obtained after zero field cooling the samples to $2 \mathrm{~K}$ from a temperature above the $T_{C}$ of the samples. Flux jumps [20] are observed in the field dependence of magnetization of butt joint and step joint niobium materials at $2 \mathrm{~K}$, which were absent at $4 \mathrm{~K}$. The occurrence of such flux jumps has been reported earlier in niobium materials [21]. Such flux jumps, however, are not very relevant to the context of the present study. The estimated value of $H_{P}$ in these samples is $\sim 1650 \mathrm{Oe}$ $(\sim 1700$ Oe) for the step joint (butt joint) niobium sample. For the unwelded and electropolished niobium sample [Fig. 9(c)], the value of $H_{P}$ is $\sim 1700$ Oe. These values of $H_{P}$ are quite close to the expected value of $H_{C 1} \sim 1800 \mathrm{Oe}$ (at $T=2 \mathrm{~K}$ ) in good quality samples of niobium [9]. These results clearly indicate that the intrinsic limit of accelerating gradient in the TM class cavities is unlikely to be influenced significantly by the process of electron beam welding. This statement, however, does not take into account the mechanical defects or debris which might arise during a bad welding process. Such extrinsic defects can lower the performance of a SCRF cavity very drastically.

The upper critical field $\left(H_{C 2}\right)$ of a type-II superconductor is not an important parameter directly in the context of the intrinsic limit of the accelerating gradient in an SCRF cavity. However $H_{C 2}$ determines the number of flux lines trapped in a SCRF cavity, when the cavity is cooled to liquid helium temperature in the presence of earth's magnetic field; these trapped flux lines can be a source of

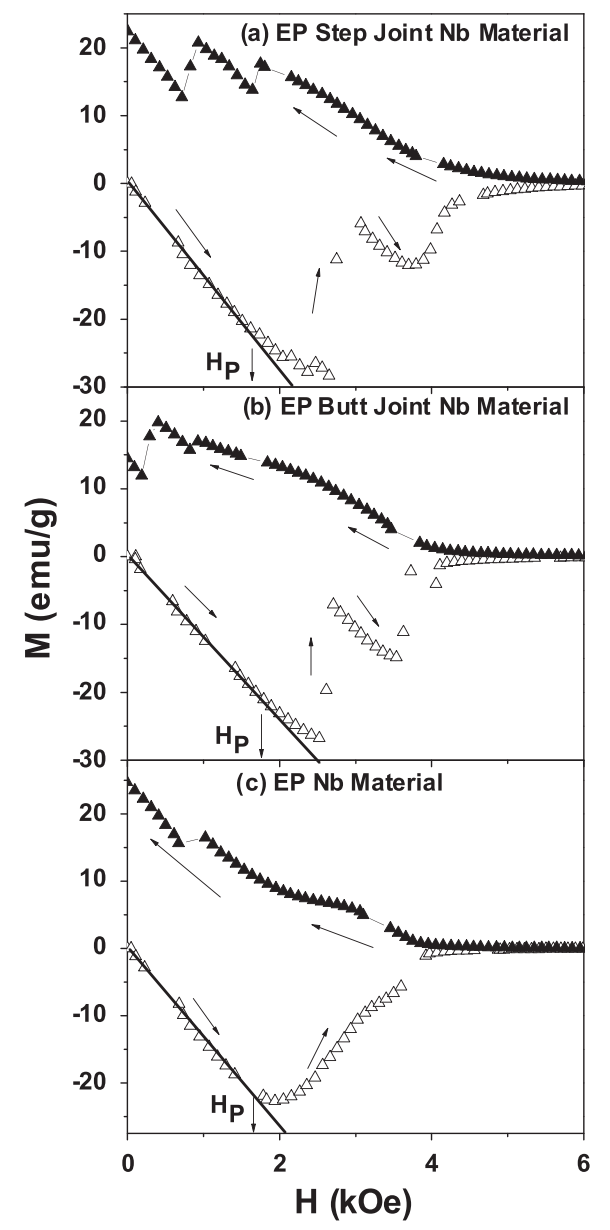

FIG. 9. Isothermal $M$ versus $H$ plots of (a) electron beam welded and electropolished niobium step joint (top), (b) electron beam welded and electropolished niobium butt joint (middle), and (c) electropolished as-received niobium (bottom), starting from the zero field cooled state at $2 \mathrm{~K}$.

dissipation in the SCRF cavity in the presence of the $\mathrm{rf}$ field. In our present study of niobium materials $H_{C 2}$ can be estimated primarily as the crossover field from the diamagnetic to paramagnetic state, and the value of $\mathrm{H}_{\mathrm{C} 2}$ estimated in this procedure is around $4500 \mathrm{Oe}$ at $T=4 \mathrm{~K}$. Using the known relation $H_{C} \approx \sqrt{\left(H_{C 2} \times H_{P}\right)}$ [22], this value of $H_{C 2}$ would lead to a value thermodynamical critical field $H_{C}$ (or superheating field limit $H_{\mathrm{SH}}$ ) $=2500$ Oe at $T=4 \mathrm{~K}$. Such a large value of $H_{C}$ or $H_{\mathrm{SH}}$ is not expected in niobium materials at $T=4 \mathrm{~K}$, which in turn puts serious doubt on the estimated value of $H_{C 2}$. On careful inspection of Figs. 8(a)-8(c), a distinct knee is observed in the $M-H$ curve in all the niobium samples around 3000 Oe with a long tail leading to the diamagnetic to paramagnetic crossover around 4500 Oe. The field where this knee in the $M-H$ curve is observed, tallies with the value of $H_{C 2}$ in niobium materials reported in the literature. We tentatively attribute this relatively long tail in the $M-H$ curve before reaching the paramagnetic normal state to a surface superconducting sheath. 


\section{CONSIDERATIONS ON THE PERFORMANCE OF NIOBIUM QWRS}

From the above study of the superconducting properties of the niobium materials, it is expected that SCRF resonators fabricated with such niobium materials would not be limited by the magnetic field in reaching high gradients, close to the intrinsic limit. We shall test this hypothesis by comparing the results from the present measurements with accelerating gradients achieved in the QWRs (Fig. 2) installed in the superconducting linac at IUAC. The QWRs have been fabricated in-house using the infrastructure set up for this purpose [23]. The niobium resonator is closely jacketed in an outer stainless steel vessel. The annular region between the niobium resonator and the stainless steel vessel, and the hollow central conductor (coaxial line) holds the liquid helium needed for cooling. The resonator is quite complex to fabricate and involves more than 30 electron beam welds. The critical welds in the high magnetic field region of the resonator are indicated in Fig. 2.

The QWR structure has been modeled using CSTMicrowave Studio (MWS) [24], which is a 3D electromagnetic simulator based on the finite integration technique. The electromagnetic parameters of the resonator have been measured using the standard bead pull technique [25] and these agree with the MWS simulated values very well. In Table I the relevant parameters of the resonator are given.

A dozen QWRs have been fabricated indigenously, out of which six resonators have been tested at $4.2 \mathrm{~K}$. The QWRs were electropolished to remove $\sim 150 \mu \mathrm{m}$ thick layer from the niobium surface during their fabrication, following the procedure briefly described in Sec. III. Subsequently, after completion of the resonator assembly, they were electropolished to remove another 50-75 $\mu \mathrm{m}$ before the cold test. After this EP, the resonators were thoroughly rinsed with deionized water followed by warm water $\left(\sim 50^{\circ} \mathrm{C}\right)$ ultrasonic cleaning for 90 minutes. After loading in the cryostat the resonators were baked in vacuum $\left(\sim 10^{-6} \mathrm{mbar}\right)$ at $\sim 115^{\circ} \mathrm{C}$ for 48 hours. The cryostats have magnetic shielding (CO-NETIC alloy) installed to shield the earth's magnetic field.

In cold tests at $4.2 \mathrm{~K}$, the QWRs have typically achieved 5-6 MV/m accelerating gradients with $6 \mathrm{~W}$ rf input power

TABLE I. Relevant parameters of the quarter wave resonator used in the superconducting linac at IUAC.

\begin{tabular}{lc}
\hline \hline Resonator parameter & Value \\
\hline Frequency $f$ & $97 \mathrm{MHz}$ \\
Synchronous velocity $\beta_{0}$ & 0.081 \\
Energy content $U_{0} @ E_{a}=1 \mathrm{MV} / \mathrm{m}$ & $110 \mathrm{~mJ}$ \\
$B_{\text {peak }} @ E_{a}=1 \mathrm{MV} / \mathrm{m}$ & $132 \mathrm{Oe}$ \\
$E_{\text {peak }} @ E_{a}=1 \mathrm{MV} / \mathrm{m}$ & $3.9 \mathrm{MV} / \mathrm{m}$ \\
Geometry factor $Q R_{s}$ & 15.1 \\
\hline \hline
\end{tabular}

in continuous wave $(\mathrm{CW})$ mode. This is the typical $\mathrm{CW}$ power level at which the resonators operate in the linac. The maximum $\mathrm{CW}$ gradients achieved before quenching were around 6-7 MV/m. To achieve these high $\mathrm{CW}$ gradients, high power pulse processing (HPP) and helium pulse processing [4] has been performed on the QWRs. During HPP processing the QWRs have achieved $7-8.5 \mathrm{MV} / \mathrm{m}$ accelerating gradients.

In Fig. 10, the performance of one of the resonators among the IUAC QWRs, that has achieved the highest gradient in the $\mathrm{CW}$ mode at $4.2 \mathrm{~K}$, is shown. The resonator could achieve $6.4 \mathrm{MV} / \mathrm{m}$ with $6 \mathrm{~W}$ rf input power. $Q$ slope was observed beyond $E_{a}=4.5 \mathrm{MV} / \mathrm{m}$ and the maximum accelerating gradient of $7 \mathrm{MV} / \mathrm{m}$ was achieved with $13.5 \mathrm{~W}$ of rf power. Beyond this gradient the resonator could not be operated in $\mathrm{CW}$ mode. At this gradient the peak magnetic field is 924 Oe. During HPP processing, however, the resonator was operated in pulsed mode with a duty cycle of $25 \%$, at the maximum gradient of $8.8 \mathrm{MV} / \mathrm{m}$ for two hours. At this gradient the estimated peak magnetic field is around 1160 Oe. The reported best peak values of magnetic field at $4.2 \mathrm{~K}$ in different quarter wave resonator designs operating at various laboratories range from 880 to 1180 Oe [26]. The highest value of 1180 Oe has been reported on the $81 \mathrm{MHz}(\beta=0.041)$ QWR at Michigan State University [26].

Comparison of the performance of the IUAC QWR with the results of measurements of superconducting properties of the niobium materials presented in Fig. 8 suggests that the peak magnetic field achieved at the highest accelerating gradient of $8.8 \mathrm{MV} / \mathrm{m}$ is close to the measured values of $H_{P}$ in electron beam welded and electropolished samples of niobium. Unlike the TM class cavities, in the TEM class resonators that are jacketed with an outer vessel, it is very difficult to attach thermometers at various locations of the resonator (Fig. 2) to identify the hot spots that may lead to thermal breakdown. In the absence of a thermal map of the

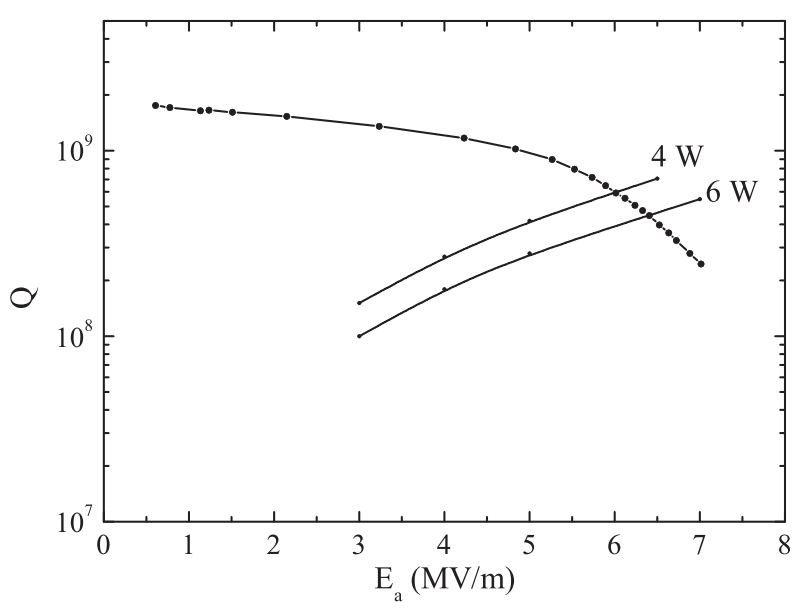

FIG. 10. Resonator $Q$ as a function of the accelerating gradient $E_{a}$ at $4.2 \mathrm{~K}$. 
resonator, it is not possible to pin down the mechanism of breakdown of the field gradient. The difference in the two values of the peak magnetic fields between the niobium samples and the QWR could possibly be on two accounts. First, while the cold test of the QWR is performed at $4.2 \mathrm{~K}$, the $H_{P}$ in the niobium materials was measured at $4 \mathrm{~K}$. Second, the maximum gradient achieved in the pulsed mode is substantially more than that in the $\mathrm{CW}$ mode (by about 25\%). This observation along with the $Q$ slope seen in Fig. 10 provides indirect evidence of thermal breakdown before the resonator could reach the intrinsic limit of the magnetic field.

It is also to be noted that the BCS surface resistance of a superconductor $\left(R_{\mathrm{BCS}}\right)$ actually determines the quality factor $(Q)$ of the SCRF cavity. Both the temperature and field dependence of $R_{\mathrm{BCS}}$ will also play important roles in the observed $Q$ drop often seen near the limit of accelerating gradient. Hence, a study of $R_{\mathrm{BCS}}$ in the step and butt joint EBW region of niobium materials will also provide valuable information on the expected performance of a SCRF cavity.

\section{CONCLUSIONS}

The superconducting properties of high purity niobium materials, which have undergone step joint and butt joint electron beam weldings (EBW), have been studied. The same materials have been used for making superconducting quarter wave resonators (QWR), which involve many such step and butt joint EBWs. We have compared the results from the magnetization measurements on electropolished as-received samples, with welded and electropolished samples to bring out differences, if any, brought about by the EBW process. It is shown that the superconducting properties of the niobium materials are not degraded due to the process of EBW. No significant difference was observed between the butt joint and step joint welded samples. The cold test of a QWR reveals that the limiting accelerating gradient is close to the intrinsic limit of accelerating gradient as predicted from the measurement of the field for first flux-line penetration $\left(H_{P}\right)$ in the superconducting niobium materials. It also suggests that the measurement of $H_{P}$ can be used for effective characterization of niobium material and various processes employed in the fabrication of SCRF cavities.

\section{ACKNOWLEDGMENTS}

The authors would like to thank K. K. Mistri for his help in the electron beam welding of the niobium samples, S. S. K. Sonti for his help in electropolishing of the samples, and J. Sacharias for his help in preparing the drawings.

[1] L. M. Bollinger, in Proceedings of XIX International Linac Conference, LINAC98, Chicago, USA (NTIS, Springfield,
VA, 1998), p. 3 [http://accelconf.web.cern.ch/AccelConf/ 198/PAPERS/MO1001.PDF].

[2] S. D. Holmes, in Proceeding of 1st International Particle Accelerator Conference, IPAC 2010, Kyoto, Japan, p. 1299 [http://accelconf.web.cern.ch/AccelConf/IPAC10/ papers/tuyra01.pdf].

[3] M.P. Kelly, in Proceedings of the 2006 Linear Accelerator Conference, LINAC06, Knoxville, TN, USA, p. 23 [http://accelconf.web.cern.ch/AccelConf/106/ PAPERS/MO3002.PDF].

[4] Hasan Padamsee, Jens Knobloch, and Tom Hays, $R F$ Superconductivity for Accelerators (Wiley-VCH Verlag $\mathrm{GmbH} \&$ Co. KGaA, Weinheim, 2008), 2nd ed.

[5] B. Aune et al., Phys. Rev. ST Accel. Beams 3, 092001 (2000).

[6] W. Singer, D. Proch, and A. Brinkmann, in Proceeding of 8th Workshop on RF Superconductivity, Abano Terme (Padova), Italy, 1997, p. 850 [http://accelconf.web .cern.ch/AccelConf/SRF97/papers/srf97d07.pdf].

[7] P. Kneisel, B. Lewis, and T. Turlington, in Proceedings of 6th Workshop on RF Superconductivity, CEBAF, Newport News, Virginia, USA, 1993, p. 628 [http:// accelconf.web.cern.ch/AccelConf/SRF93/papers/srf93i09 .pdf].

[8] G. Ciovati, P. Kneisel, and G. Myneni, in Proceedings of 11th Workshop on RF Superconductivity, Lubeck, Germany, 2003, p. 562 [http://accelconf.web.cern.ch/ AccelConf/SRF2003/papers/weo14.pdf].

[9] D. K. Finnemore, T.F. Stromberg, and C. A. Swenson, Phys. Rev. 149, 231 (1966).

[10] S. B. Roy, G. R. Myneni, and V. C. Sahni, Supercond. Sci. Technol. 21, 065002 (2008).

[11] T. Hays, H. Padamsee, and R. W. Roth, in Proceedings of 7th Workshop on RF Superconductivity, Gif-sur-Yvette, France, 1995, p. 437.

[12] P. M. Chaikin and T.C. Lubensky, Principles of Condensed Matter Physics (Cambridge University Press, Cambridge, England, 1998).

[13] G. Ciovati and P. Kneisel, Phys. Rev. ST Accel. Beams 9, 042001 (2006).

[14] S. B. Roy, G. R. Myneni, and V. C. Sahni, Supercond. Sci. Technol. 22, 105014 (2009).

[15] L. Lilje et al., Nucl. Instrum. Methods Phys. Res., Sect. A 524, 1 (2004).

[16] S. Casalbuoni et al., Nucl. Instrum. Methods Phys. Res., Sect. A 538, 45 (2005).

[17] H. Diepers, O. Schmidt, H. Martens, and F. S. Sun, Phys. Lett. 37A, 139 (1971).

[18] Technical notes on VSM, Quantum Design, USA [http://www.qdusa.com/sitedocs/appNotes/vsmappnote_5-09 .pdf].

[19] IsoMet Low Speed Saw, Buehler, USA [http://www .buehler.com/productinfo/precision_saws/isomet_low_speed .pdf].

[20] M. Tinkham, Introduction to Superconductivity (Dover Publications, New York, 1996).

[21] M. Levy, R. Kagiwada, F. Carsey, and I. Rudnick, Phys. Rev. B 2, 2804 (1970).

[22] C.P. Poole, H. A. Farach, and R.J. Creswick, Superconductivity (Academic Press, New York, 1995). 
[23] Prakash N. Potukuchi, in Proceedings of 14th International Conference on RF Superconductivity SRF2009, Berlin, Germany, 2009, p. 502 [http://accelconf.web.cern.ch/ AccelConf/SRF2009/papers/thobau05.pdf].

[24] Computer Simulation Technology, Darmstadt, Germany [http://www.cst.com].
[25] L.C. Maier and J.C. Slater, J. Appl. Phys. 23, 68 (1952).

[26] A Facco, TESLA Technology Collaboration Meeting, 2010, Fermi National Accelerator Laboratory, USA [http://conferences.fnal.gov/ttc10/]. 